An official journal of the / Un journal officiel de la "Société Sénégalaise de Cancérologie" (SOSECAN)

Journal homepage: www.africanjournalofoncology.com

Original article / Article original

DOI: https://doi.org/I0.54266/ajo.I.I.3.DRUG8652

\title{
Childhood glioblastomas at Fann's neurosurgery department in Dakar
}

\section{Les glioblastomes de l'enfant au service de neurochirurgie de Fann à Dakar}

EHCN. Sy', M. Thioub', M. Mbaye', Y. Cissé', AB. Thiam', MC. Ba', SB. Badiane'.

I Service de neurochirurgie, Centre Hospitalier National Universitaire de Fann (CHNU), Sénégal.

INTRODUCTION: Glioblastoma (GBM) is a tumor usually found in adults. However, it is rare in pediatrics. Their prognosis is uniformly pejorative and the guidelines for management are still not codified. we have realized a retrospective study in order to search clinical and pathological features as well as any prognostic factors. PATIENTS AND METHODS: This is a retrospective study of 8 children with GBM confirmed by histology, collected at the neurosurgery department of the Fann National University Hospital Center, from January I, 2016 to December 3I, 2018. RESULTS: The annual frequency was 2.6 cases. There was a male predominance with a sex ratio of I.6. The time limit consultation time was three and a half months. The main reason for consultation was intracranial hypertension syndrome (HTIC), found in $62.5 \%$ of cases. The region most affected was the parietal lobe $(n=4)$. The mean time to surgical management was 2.3 months. Excisional surgery resulted in macroscopically complete resection in half of the cases. The histological study found eight cases of WHO grade IV glioblastoma. Only one patient had received adjuvant radiochemotherapy. The six months' mortality was $85.7 \%$ with a median survival of 6.5 months. CONCLUSION: Glioblastomas remain a rarity in children. Multimodal treatment combining excisional surgery, chemo and radiotherapy remains the guarantee for a better prognosis.

KEYWORDS: Glioblastoma; Children; Dakar.

INTRODUCTION : Le glioblastome (GBM) est une tumeur généralement de l'adulte. Cependant, il est rare en pédiatrie. Leur pronostic est uniformément péjoratif et les directives de prise en charge restent encore non codifiées. Nous avons réalisé une étude rétrospective afin d'en rechercher les particularités cliniques et pathologiques ainsi que les éventuels facteurs pronostiques. PATIENTS ET METHODES : II s'agit d'une étude rétrospective portant sur 8 enfants porteurs de GBM confirmé à l'histologie, colligés au service de neurochirurgie du Centre Hospitalier National Universitaire de Fann, du I er janvier 2016 au 3 I décembre 2018. RESULTATS : La fréquence annuelle était de 2,6 cas. On notait une prédominance masculine avec un sex-ratio de I,6. Le délai de consultation moyen était de 3 mois et demi. Le motif de consultation principal était le syndrome d'hypertension intracrânienne (HTIC) retrouvé chez $62,5 \%$ des cas. La région la plus concernée était le lobe pariétal $(n=4)$. Le délai moyen de prise en charge chirurgicale était de 2,3 mois. La chirurgie d'exérèse a permis une résection macroscopiquement complète dans la moitié des cas. L'étude histologique avait retrouvé huit cas de glioblastome classé grade IV de l'OMS. Un seul patient avait bénéficié d'une radio-chimiothérapie adjuvante. Le taux de mortalité à 6 mois était de $85,7 \%$ avec une médiane de survie de 6,5 mois. CONCLUSION : Les glioblastomes demeurent une rareté chez l'enfant. Le traitement multimodal associant chirurgie d'exérèse, chimio- et radiothérapie reste le garant pour un meilleur pronostic.

MOTS-CLES : Glioblastome ; Enfant ; Dakar.

\section{INTRODUCTION}

Les tumeurs cérébrales sont les tumeurs solides les plus fréquentes en pédiatrie. Elles constituent la première cause de morbidité et de décès liés au cancer chez l'enfant [I]. Le glioblastome (GBM) est la tumeur cérébrale primitive maligne la plus fréquente de l'adulte. Chez l'enfant, il est rare et représente environ $5 \%$ à $10 \%$ des tumeurs cérébrales pédiatriques [2, 3]. En Afrique subsaharienne, du fait de sa rareté, l'incidence des GBM de l'enfant reste inconnue, en particulier au Sénégal, où aucune étude n'a été réalisée jusqu'à ce jour. L'objectif de notre étude était de rechercher les particularités cliniques et pathologiques ainsi que les éventuels facteurs pronostiques des glioblastomes de l'enfant au service de neurochirurgie du Centre Hospitalier National Universitaire (CHNU) de Fann.

\section{PATIENTS ET METHODES}

Nous avons réalisé une étude rétrospective, analytique, transversale portant sur 8 patients âgés de moins de 16 ans porteurs de tumeurs cérébrales et colligés sur une période de 3 ans allant du $I^{\mathrm{er}}$ janvier 2016 au 3 I décembre 2018 au service de neurochirurgie du CHNU de Fann. Ont été inclus dans notre étude, tous les cas pédiatriques chez lesquels le diagnostic formel de glioblastome ou astrocytome malin grade IV (OMS, 2007) a été posé après étude histologique. Les cas suspectés sur la base de l'imagerie cérébrale (tomodensitométrie (TDM), ou TDM et imagerie par résonnance magnétique (IRM) ou IRM) et qui n'ont pas été opéré, n'ont pas été inclus. L'autorisation pour mener ce travail a été obtenue au sein du comité d'éthique du CHNU de Fann de Dakar. 


\section{RESULTATS}

Notre population d'étude était composée de huit cas pédiatriques de glioblastomes sur les 66 cas de tumeurs cérébrales sus-tentorielles opérées chez les enfants. Il s'agissait de cinq garçons et trois filles, l'âge moyen était de II,2 ans avec des extrêmes de huit et 15 ans. Le délai moyen de consultation était de trois mois et 15 jours avec des extrêmes de 28 jours et cinq mois. Nous avions noté des antécédents familiaux de tumeurs gliales chez deux de nos patients dont un cas issu d'une fratrie de neuf enfants dont il est le dernier avec six décès dans la famille dont deux cas de gliomes de haut grade décédés dans notre service. Le motif de consultation était un syndrome d'hypertension intracrânienne dans $62,5 \%$ des cas $(n=5)$, plus rarement un déficit moteur $(n=2)$, une épilepsie $(n=2)$ ou des troubles visuels à type de baisse de l'acuité visuelle $(n=I)$ (Tableau I).

Tableau I : Motifs de consultation

\begin{tabular}{|lll|}
\hline Signes & Nombre & Pourcentage (\%) \\
\hline HTIC & 5 & 62,5 \\
Epilepsie & 2 & 25 \\
Déficit moteur & 2 & 25 \\
BAV & 1 & 12,5 \\
\hline
\end{tabular}

HTIC : hypertension intracrânienne

La TDM cérébrale a été réalisée chez tous les patients (I00\%) mettant en évidence dans la majorité des cas une lésion hétérogène lobaire entourée d'œdème péri-lésionnel important et un effet de masse sur les structures médianes (Figure I).
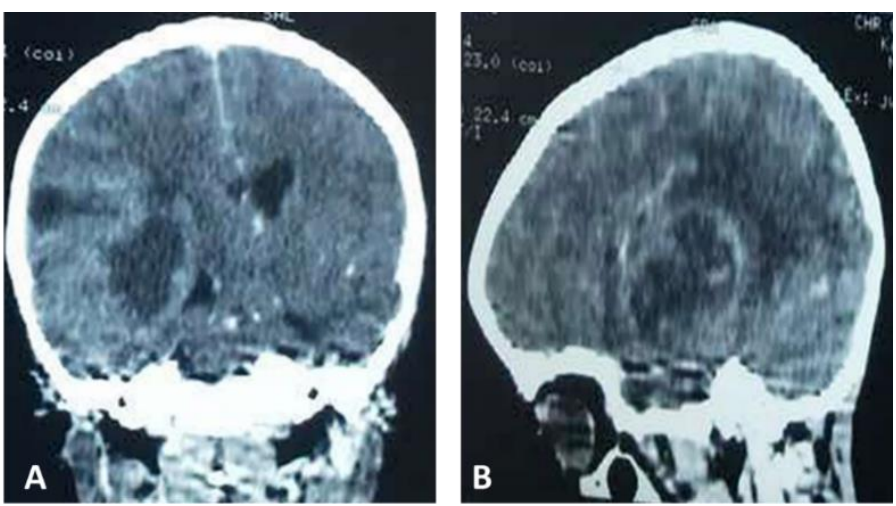

Figure I : Tomodensitométrie cérébrale injectée en coupes coronale $(A)$ et sagittale (B) montrant un glioblastome temporal droit à double composante kystique et charnue prenant le contraste de façon hétérogène associé à un œdème péri-lésionnel important. La lésion exerce un effet de masse sur les structures médianes, avec début d'engagement temporal.

\section{DISCUSSION}

Le GBM est une tumeur rare en pédiatrie. Son incidence est de l'ordre de 0,6/100.000 chez les enfants et adolescents âgés de zéro à 19 ans [4]. Dans notre série, la fréquence annuelle était 2,6 et les GBM représentaient $12 \%$ des tumeurs cérébrales sus-tentorielles opérées chez les enfants. Ces chiffrent sont élevées par rapports aux données de la littérature ou la fréquence varie entre $5 \%$ et $9 \%$ [5-8]. Ces chiffrent s'expliquent par le fait que notre centre reste le service de référence pour la neurochirurgie pédiatrique dans tout le Sénégal et dans la sous-région. La vulgarisation de l'imagerie dans le territoire contribue aussi à l'augmentation de cette fréquence. L'âge moyen était de $1 \mathrm{I}, 2$ ans ce qui corrobore avec la littérature [8-10]. La prédominance masculine retrouvée est une donnée classique de la littérature [5].

Les caractéristiques histologiques des GBM sont identiques quel que soit l'âge du patient ou la localisation de la tumeur, mais leurs caractéristiques génétiques diffèrent [II]. Dans notre étude, aucune analyse génétique n'a été faite par manque de plateau technique. Des preuves croissantes suggèrent que les patients atteints de L2-HGA sont prédisposés aux néoplasmes cérébraux [12]. L'acidurie L2-hydroxyglutarique (L2-HGA) est une maladie neurométabolique autosomique récessive rare caractérisée par l'accumulation de L2hydroxyglutarate (L2-HG), un oncométabolite potentiel entraînant un risque important de tumeurs cérébrales au cours de la vie. Il est ainsi connu que plus de $65 \%$ des GBM secondaires sont associés à une mutation du gène TP53 alors qu'elle est retrouvée dans moins de $28 \%$ des GBM primaires. Les mutations du gène IDHI (isocitrate déshydrogénase) sont fréquentes dans les GBM secondaires (>80\%) mais sont absentes ou rarement décelées dans les GBM primaires $(<10 \%)$. Parmi les autres facteurs : mutation du gène TP53, mutations du gène IDHI [I2, I3].

Le délai moyen de consultation était de trois mois et demi. Les tumeurs de haut grade se caractérisent par une évolution très rapide. Certains auteurs rapportent un délai plus court [14]. La symptomatologie clinique des GBM de l'enfant n'est pas spécifique. Le motif de consultation prédominant dans notre étude était un syndrome d'hypertension intracrânienne dans $57.1 \%$ des cas. Nos chiffrent s'accordent avec la littérature [5, 14].

Les glioblastomes sont plus fréquents à l'étage supratentoriel [14]. Dans notre série, tous les glioblastomes sont situés dans ce compartiment. De nombreux auteurs ont rapportés dans leur série, des localisations sous tentorielles bien que rare. [7, $9,10]$. Cependant, si la plupart des auteurs s'accordent sur la prédominance de l'étage sus-tentoriel, la prédominance d'un lobe sur un autre ne fait pas l'unanimité.

Tous nos patients ont été opérés. L'analyse de la littérature confirme le rôle de la résection sur la survie [15]. Malgré un traitement multimodal par chirurgie, chimiothérapie et radiothérapie, les enfants atteints de GBM ont généralement un mauvais pronostic. Chez les adultes, au contraire une amélioration modeste de la survie est rapportée avec l'association du témozolomide et bevacizumab à la radiothérapie conformationnelle [16] et d'autres rapportent par contre une amélioration plus significative avec le même schéma thérapeutique similaire lorsque qu'il est entrepris précocement après la chirurgie [16]. 85,7\% des patients de notre série ont succombé. La médiane de survie était de 6,5 mois. Des auteurs rapportent une survie médiane qui varie de 13 à 20 mois après une radiothérapie complémentaire $[9,10]$. Dans notre série, un seul patient a reçu une radiothérapie complémentaire. L'absence de traitement complémentaire, en l'occurrence la radiothérapie et la chimiothérapie explique notre courte médiane de survie. Mais aussi la prise en charge retardée y contribue de beaucoup.

\section{CONCLUSION}

Les glioblastomes demeurent une rareté chez l'enfant. Les recherches de cette dernière décennie ont mis en évidence le 
rôle primordial des processus épigénétiques dans la formation et la progression tumorales. La place de l'analyse génétique et moléculaire est plus que primordiale pour mieux cerner cette affection. Le traitement multimodal associant chirurgie d'exérèse, chimiothérapie et radiothérapie reste le garant pour un meilleur pronostic.

\section{CONFLITS D'INTERET}

Les auteurs n'ont déclaré aucun conflit d'intérêts.

\section{REFERENCES}

I. Bauchet $L$ et al. Clinical epidemiology for childhood primary central nervous system tumors J Neurooncol 2009; 92 (I):87-98.

2. Friedman GK, Spiller SE, Harrison DK, Fiveash JB., Reddy AT. Treatment of Children With Glioblastoma With Conformal Radiation, Temozolomide, and Bevacizumab as Adjuncts to Surgical Resection, J Pediatr Hematol Oncol 2013; 35:e I23-el 26.

3. Koob M, Girard N. Cerebral tumors: Specific features in Children; Diagnostic and Interventional Imaging 2014;95:965-983.

4. Verreault M, Delattre JY, Ahmed Idbaih A. Les tumeurs gliales diffuses de l'adulte : hétérogénéité génétique et implications thérapeutiques. Med Sci 2012;28:8I2-6.

5. Ibahioin $\mathrm{K}$, Ahanogbe $\mathrm{KMH}$, Dianka MB, Bertal $\mathrm{A}$, Chellaoui A, Hilmani S et al. Glioblastome de l'enfant, analyse descriptive au CHU IBN Casablanca, Maroc; Rev Maroc Neurochir 2016 ; I (I) : I- I 2.

6. Harmouch et al. Epidemiology of pediatric primary tumors of the nervous system: a retrospective study of 633 cases from a single Moroccan institution. Neurochirurgie 2012;58(I): I4-8.

7. Das, KK, Mehrotra A, Nair AP et al. Childs Nerv Syst 2012;28: 2055.

8. Suri V, Das P, Pathak P, Jain A, Sharma MC, Borkar SA, Suri A, Gupta D, Sarkar C. Pediatric glioblastomas: a histopathological and molecular genetic study. Neuro Oncol 2009; I I (3):274-80.

9. Nikitović, M., Stanić, D., Pekmezović, T. et al. Childs Nerv Syst 2016;32: 97.

10. Perkins SM, Rubin JB, Leonard JR, Smyth MD, El Naqa I, Michalski JM, Simpson JR, Limbrick DL, Park TS, Mansur DB Glioblastoma in children: a singleinstitution experience. Int J Radiat Oncol Biol Phys 20II; 80(4):III7-2I.

II. Faury D, Nantel.A, Dunn SE et al Molecular profiling identifies subsgroups of pediatric glioblastoma and shows increased YB-I expression in tumors, Clin Oncol 2007;25: I 196-268.

12. Haliloglu GI, Jobard F, Oguz KK, Anlar B, Akalan N , Coskun T, Sass JO, Fischer J, Topcu M. Acidurie L-2-hydroxyglutarique et tumeurs cérébrales chez les enfants porteurs de mutations du gène L2HGDH: résultats de la neuro-imagerie. Neuropédiatrie 2008 ; 39 (2): $119-22$.

13. Ohgaki H. Genetic pathways to glioblastomas. Neuropathology 2005; 25(I):I-7.

14. Artico M, Cervoni L, Celli P, Salvati M, Palma L (1993) Supratentorial glioblastoma in children: a series of 27 surgically treated cases. Childs Nerv Syst 9:7-9
15. Menei P, Metellus P. Surgical treatment of glioblastomas, Neurochirurgie 2010; 56(6):477-482.

16. Friedman GK, Spiller SE, Harrison DK, Fiveash JB, Reddy AT. Treatment of children with glioblastoma with conformal radiation, temozolomide, and bevacizumab as adjuncts to surgical resection. J Pediatr Hematol Oncol 2013;35(3): I23-6. 